Rapport - Société canadienne d'histoire de l'Église catholique

\title{
Les débuts de Saint-Sulpice à Montréal (1657-1688)
}

\section{Jean Langis}

Volume 31, 1964

URI : https://id.erudit.org/iderudit/1007341ar

DOI : https://doi.org/10.7202/1007341ar

Aller au sommaire du numéro

Éditeur(s)

La Société canadienne d'histoire de l'Église catholique

ISSN

0318-6148 (imprimé)

1927-7075 (numérique)

Découvrir la revue

Citer cet article

Langis, J. (1964). Les débuts de Saint-Sulpice à Montréal (1657-1688). Rapport -

Société canadienne d'histoire de l'Église catholique, 31, 15-22.

https://doi.org/10.7202/1007341ar

Tous droits réservés @ C La Société canadienne d'histoire de l'Église catholique, 1965
Ce document est protégé par la loi sur le droit d'auteur. L'utilisation des services d’Érudit (y compris la reproduction) est assujettie à sa politique d'utilisation que vous pouvez consulter en ligne.

https://apropos.erudit.org/fr/usagers/politique-dutilisation/ 


\title{
Les débuts de Saint-Sulpice à Montréal (1657-1688)
}

\begin{abstract}
Histoire abrégée (de 1657 à 1688) des premiers fils de $M$. Olier à Montréal : M. de Queylus, premier supérieur du Séminaire; M. Souart, premier curé missionnaire de Ville-Marie; M. Galinier, son vicaire; M. d'Allet, secrétaire de M. de Queylus. L'auteur raconte aussi brièvement la mort tragique de $M M$. Vignal et LeMaître, et termine par quelques notes rapides sur $M$. Gilles Pérot, premier «curé d'office •t sur M. Dollier de Casson.
\end{abstract}

Le 29 juillet 1657, déharquent à Québec, en route pour Montréal, quatre ecclésiastiques français, envoyés par M. Olier, fondateur de SaintSulpice. Trois d'entre eux sont prêtres : MM. Gabriel de Queylus, Gabriel Souart et Dominique Galinier; l'autre est seulement diacre : M. Antoine d'Allet.

Je n'ai pas voulu exposer la genèse de ce fait historique, par exemple comment $\mathrm{M}$. Olier a été amené à poser ce geste : il y faudrait déjà une longue conférence. Je veux seulement, partant du fait lui-même, vous présenter les quatre personnages et vous décrire le travail qu'ils ont accompli ici, depuis leur arrivée, en 1657, jusqu'au départ du dernier d'entre eux, en 1688. Peut-être introduirai-je, en cours de route, tel ou tel autre de leurs confrères plus important venu travailler à Montréal entre ces deux dates.

Gabriel de Thubières de Lévy Queylus est né en 1612, dans le Rouergue, d'une famille ancienne et fortunée. Dès l'âge de 11 ans, il est abbé de Loc-Dieu. Etudes sérieuses jusqu'au doctorat en théologie. En 1641, à 29 ans, il est reçu comme séminariste à Vaugirard, où M. Olier travaille à établir un séminaire, après un essai infructueux à Chartres. L'année suivante, $M$. Olier est nommé curé de la paroisse de Saint-Sulpice, à Paris. Il y transfère son séminaire de Vaugirard, qui devient le Séminaire de Saint-Sulpice - d'où le nom de Sulpiciens donné aux prêtres, groupés en société, qui le dirigent. Monsieur de Queylus y est ordonné en 1645 et, presque aussitôt, il " entre à SaintSulpice ». Il est donc un des premiers fils de M. Olier. Il est aussi son homme de confiance. Lorsque M. Olier fonde les séminaires de Nantes (1649), de Viviers (1650), de Clermont (1653), qui place-t-il à la tête de ces maisons ? M. de Queylus. Et lorsque, enfin, M. Olier décide de fonder à Montréal, il rappelle de Clermont M. de Queylus et le nomme supérieur du séminaire de Montréal. Monsieur de Queylus est bien connu comme prédicateur de missions populaires et il est estimé de tous. On parle même de lui à l'Assemblée du clergé de France, le 10 janvier 1657 : avec l'appui de Godeau, évêque de Vence, il est dési- 
gné pour l'évêché du Canada, mais sa nomination est bloquée à la Cour. Monsieur de Queylus partira donc, non comme évêque, mais comme vicaire général de François de Harlay de Champvallon, archevêque de Rouen, et sa juridiction s'étendra à tout le pays, ce qui l'opposera d'abord aux Jésuites, puis à $\mathrm{M}^{\mathrm{gr}}$ de Laval. Monseigneur Olivier Maurault a raconté ce conflit dans un article excellent, paru dans le Rapport de votre Société pour 1955-1956, sous le titre : "Saint-Sulpice et le Canada : l'imbroglio Queylus-Laval. »

Monsieur Gabriel Souart est plus vieux que son supérieur d'un an ou deux. Il est né à Paris, en 1610 ou 1611. Il est moins a voyant " que M. de Queylus, mais combien attachant! En 1646, il passe subitement de l'étude de la médecine et des fiançailles à l'état ecclésiastique : encore une vocation tardive... Avec son frère Louis, il s'était joint à M. Olier. Leur père était premier apothicaire du duc d'Orléans et ami de M. Olier. Fortune considérable. Les deux frères Souart, de concert avec leurs parents, avaient donné à M. Olier de quoi jeter les fondements du premier Séminaire de Saint-Sulpice. Bachelier en droit canon, Gabriel Souart est ordonné prêtre en 1650. Il part pour Montréal avec le titre de curé. Le Canada lui est déjà connu : il est le neveu d'un des premiers missionnaires, le père Joseph Le Caron, récollet.

Monsieur Dominique Galinier ne nous retiendra pas longtemps. D'abord, parce que les documents sur lui sont d'une discrétion décourageante, ensuite, parce qu'il a plutôt travaillé aux missions indiennes qu'à Montréal même. Il naît en 1616, au diocèse de Mírepoix, entre à Saint-Sulpice de Paris en 1645 et est ordonné en 1650 ou 1651. Il vient à Montréal comme vicaire. Il a 41 ans.

Le quatrième membre du groupe des fondateurs est $M$. Antoine d'Allet. Né vers 1634, il n'était que diacre en 1657. Il venait au Canada comme secrétaire de M. de Queylus. La Liste chronologique des Evêques et des Prêtres, publiée à Québec en 1834, indique le 15 août 1659 comme date de son ordination sacerdotale. C'est possible, puisque M. de Queylus et lui se trouveront alors à Québec, où vient d'arriver $\mathbf{M}^{\mathrm{gr}}$ de Laval; mais aucun document connu ne permet de l'affirmer positivement.

Nos quatre voyageurs s'embarquèrent à Saint-Nazaire (port de Nantes), le 17 mai 1657, en compagnie de M. de Maisonneuve. Le 29 juillet, ils mettaient pied à terre à l'Ile d'Orléans, puis se rendaient à Québec, où ils passèrent quelques jours. Avant de les voir à l'œuvre à Montréal, essayons de reconstituer, dans ses traits essentiels, le cadre de leur apostolat. En d'autres termes, qu'était Montréal en 1657 ?

Après quinze ans d'existence, d'une existence toujours dramatique, souvent héroïque, Montréal n'est pas autre chose qu'un village qui, très probablement, ne compte pas encore deux cents âmes. D'après le gouverneur d'Argenson, il n'y avait à Montréal, au printemps de 1659, qu'environ quarante maisons, cinquante chefs de famille et cent soixante 
personnes en tout. En 1657, il y a trente-huit enfants, de zéro à huit ans. L'année suivante, Marguerite Bourgeoys pourra commencer à faire l'école. Les maisons, en bois, sont situées les unes en face des autres, pour se protéger mutuellement, et percées de meurtrières, par où l'on peut faire feu sur les assaillants... Pas de rues, mais de simples sentiers. Pas de puits, mais seulement des citernes et des fosses. Pour église, d'abord la petite chapelle du Fort, et maintenant la chapelle de l'hôpital, avec son clocher que Jeanne Mance qualifie de a considérable ". Pas de presbytère : pendant quinze années, les Pères Jésuites qui ont desservi Montréal ont résidé au Fort, où logent encore, en ce moment, M. de Maisonneuve, la famille d'Ailleboust, Marguerite Bourgeoys, le major Lambert Closse et les soldats de la garnison. Signalons encore, vers l'est, à une certaine distance des habitations, un nouveau chantier : on travaille aux fondations de Notre-Dame de Bon-Secours, la chapelle de Marguerite Bourgeoys.

Pour la commodité du récit, il m'a paru préférable, au risque de quelques répétitions, de décrire séparément et, il va sans dire, brièvement, le travail de chacun des a fondateurs ».

Monsieur de Queylus a fait trois séjours au Canada : du 29 juillet 1657 au 22 octobre 1659; du 3 août au 22 octobre 1661; de 1668 à 1671. Des quatorze ans que dura son obédience comme supérieur du séminaire de Montréal, il en passa moins de six au Canada. Son premier séjour se partage presque également entre Québec et Montréal. Arrivé à Montréal le 12 août, il n'y reste que quelques jours. Avec ses confrères, il fut l'hôte de Jeanne Mance à l'Hôtel-Dieu. Il installe M. Souart comme curé, observe l'état de la colonie sans trop livrer ses impressions et repart pour Québec, où il arrive le 12 septembre. Il reprend pour lui-même la cure de Québec, que le supérieur des Jésuites, l'enlevant au père Poncet, venait de confier au père Pijart, libéré de celle de Montréal; il préside, à l'Hôtel-Dieu, la vêture de la première religieuse indienne, la huronne Geneviève; il établit, sur la côte de Beaupré, les églises de Sainte-Anne et de Château-Richer; il répond à une lettre de Marguerite Bourgeoys et lui enjoint de suspendre les travaux de Bon-Secours; il négocie en secret avec l'Hôtel-Dieu de Québec et envoie à Montréal deux Hospitalières Augustines, sous prétexte de les " changer d'air », mais en réalité pour les introduire dans l'hôpital de Jeanne Mance. En août 1658, après onze mois d'absence, il remonte à Montréal. De nouveau, on le retrouve à Québec, le 7 août 1659, attendant son bateau pour retourner en France. Le 28, en la fête de saint Augustin, il prêche à l'Hôtel-Dieu, durant la messe pontificale de $\mathbf{M}^{\text {gr }}$ de Laval.

Ici se place l'arrivée de deux autres Sulpiciens, dont le ministère à Montréal sera si bref et la fin si tragique : MM. LeMaître et Vignal.

Monsieur Jacques LeMaître se trouvait au Séminaire de Saint-Sulpice, à Paris, lorsque M. Olier nomma M. de Queylus et ses compagnons pour la mission de Montréal. Il s'offrait à M. Olier : il serait prêt à 
courir de toutes parts pour chercher des Sauvages; il irait même les trouver dans leur propre pays... " Vous n'en aurez pas la peine, lui dit M. Olier; ils viendront bien vous chercher eux-mêmes, et vous vous en trouverez si environné que vous ne pourrez vous échapper de leurs mains. 》 Paroles prophétiques, qui ne tarderont pas à se réaliser! En 1659, on envoie, de Paris, M. LeMaître à Ville-Marie, en compagnie de M. Guillaume Vignal. Celui-ci, avant d'entrer à Saint-Sulpice, avait vécu au Canada, où il avait rempli durant dix ans la charge de chapelain des Ursulines. C'est lui que M. de Queylus, en 1658, avait envoyé à

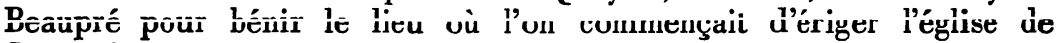
Sainte-Anne.

M. LeMaître et M. Vignal s'embarquèrent à La Rochelle en mai 1659, sur le Saint-André, qui transportait aussi Jeanne Mance et ses premières Hospitalières de Saint-Joseph, ainsi que Marguerite Bourgeoys et sa recrue, Marie Raisin. Après une traversée de soixante-sept jours, ils débarquèrent à Québec le 8 septembre. Monsieur de Queylus y était, nous l'avons vu, préparant sa rentrée en France. Il accueille ses deux confrères, les charge de l'économat de la communauté de Montréal et leur donne la mission de construire une maison pour les Sulpiciens. De plus, $M^{\mathrm{gr}}$ de Laval nomme M. Vignal confesseur à l'Hôtel-Dieu de Montréal. Marguerite Bourgeoys partit la première de Québec pour Ville-Marie et y arriva le 29 septembre. Monsieur LeMaître l'accompagnait. Jeanne Mance, ses trois religieuses et M. Vignal partirent plus tard. En route, ils croisèrent une chaloupe : c'étaient les deux Hospitalières Augustines, mandées par M. de Queylus l'année précédente, qui revenaient à Québec, avec $M$. Souart. Jeanne Mance et sa petite troupe arrivèrent à Montréal le 20 octobre, date désormais célèbre à l'HôtelDieu de Montréal. Messieurs LeMaître et Vignal eurent à peine le temps d'accomplir leur tâche. Dès 1661, ils furent tous deux massacrés par les Iroquois : M. LeMaître, le 20 août, M. Vignal, le 27 octobre. Le premier, tué sur l'île de Montréal, fut enterré dans le cimetière de l'hôpital; le second, pris par les Iroquois sur l'île à la Pierre et traîné par eux en captivité, fut tué deux jours après, et son corps n'est pas au cimetière. Messieurs LeMaître et Vignal sont les deux premiers Sulpiciens morts en service à Montréal.

Monsieur de Queylus était donc rentré en France. Monsieur de Bretonvilliers, successeur de M. Olier, et les Associés de Montréal, sachant que M. de Queylus était, par sa fortune, le principal soutien de Ville-Marie, le décident d'aller à Rome, pour obtenir l'érection canonique de la cure de Ville-Marie, qui devient alors « la paroisse de VilleMarie en l'île de Montréal ». Ayant réussi, il reprend la route du Canada. Malgré l'interdit royal qui le frappe, il gagne nuitamment Montréal, nomme M. Souart pour le remplacer comme supérieur du Séminaire, est ramené à Québec par le gouverneur d'Avaugour et reprend la mer le 22 octobre, cinq jours avant le " martyre » de M. Vignal. 
En 1663, le Séminaire de Saint-Sulpice de Paris devenait seigneur de l'île de Montréal. Monsieur Souart ne raffolait pas des fonctions de supérieur, encore moins depuis que le supérieur de Montréal représentait les seigneurs de l'île. Aussi, désirant hâter le retour de M. de Queylus, décide-t.il d'aller le chercher en France. Il s'embarque en novembre 1667, trouve $M$. de Queylus supérieur de la communauté des prêtres de la paroisse de Saint-Sulpice. Il le ramène et, avec lui, sept autres confrères. Cette fois, $\mathrm{M}^{\mathrm{gr}}$ de Laval reçut $\mathrm{M}$. de Queylus avec honneur et lui donna des lettres de grand vicaire pour Montréal.

Pendant les trois ans de son dernier séjour au Canada, on voit que le grand vicaire cherche plutôt à se faire oublier. Mais il ne reste pas inactif. « Montréal avait de grands besoins, et seul un supérieur opulent comme M. de Queylus pouvait y suffire " $\left(\mathrm{M}^{\mathrm{gr}}\right.$ Olivier Maurault, op. cit.). Monsieur de Queylus prodigue ses bienfaits : il inaugure (1668) les missions lointaines, que $\mathrm{M}^{\mathrm{gr}}$ de Laval avait jusque-là interdites aux Sulpiciens; il rachète de petits Indiens tombés entre les mains des Iroquois, ou d'autres prisonniers de guerre, promis à une mort cruelle; il pousse MM. Dollier de Casson et de Galinée (qu'il ne faut pas confondre avec M. Galinier) à aller évangéliser les tribus des Grands Lacs; il entreprend la fondation d'une école d'Arts et Métiers pour les Indiens du lac Saint-Louis, projette un hôpital pour les Indiens âgés ou malades; dans l'est de l'île, il fait les concessions qui amènent la fondation de la Pointe-aux-Trembles, etc. Désireux de réaliser son hôpital, il passe en France à l'automne de 1671, pour régler ses affaires de famille et réunir sa fortune. Mais il tombe malade, se retire chez les prêtres du Mont-Valérien, y languit quelques années et rentre finalement au Séminaire de Saint-Sulpice de Paris, où il meurt le 20 mars 1677.

Si l'on osait faire un reproche à M. de Queylus, ce serait celui-ci : il a été plus québécois que montréaliste (comme on disait alors), c'està-dire qu'il a eu moins de sympathie pour les communautés de Montréal que pour celles de Québec. Il était d'avis - comme $\mathrm{M}^{\mathrm{gr}}$ de Laval, d'ailleurs - que les deux communautés montréalistes (les Hospitalières de Saint-Joseph et la Congrégation Notre-Dame) auraient dû fusionner avec les Augustines et les Ursulines de Québec. En quoi il se mit carrément en contradiction avec la pensée et les engagements de $\mathrm{M}$. Olier et des Associés de Montréal, pensée et engagements qu'il connaissait fort bien, puisqu'il fut, dès le début, membre de la Société de Montréal et qu'il vécut de longues années dans l'intimité de M. Olier. En quoi, aussi, il fit inutilement souffrir ces deux femmes merveilleuses que furent Jeanne Mance et Marguerite Bourgeoys. Il n'en reste pas moins l'un des bienfaiteurs insignes de la colonie, pour laquelle il a dépensé presque toute sa fortune.

Transplanté à 46 ou 47 ans dans un pays combien nouveau, M. Souart se donna sans partage à sa nouvelle patrie, à laquelle il consacra plus de trente années de sa vie (1657-1688). Curé, " maître 
d'école * - c'est à ce titre qu'il tient le plus - supérieur ecclésiastique (1657) et confesseur (1663) de la Congrégation Notre-Dame jusqu'en 1676, aumônier de l'Hôtel-Dieu (1661-1678, 1679-1684), supérieur (intérimaire) du Séminaire (1661-1668), supérieur du Séminaire (16741676), ami fidèle et défenseur vigilant de Jeanne Mance et de ses Hospitalières, de Marguerite Bourgeoys et de sa Congrégation; père et bienfaiteur généreux - car il était fort riche - des pionniers, médecin et chirurgien - d'après sœur Morin, il aurait obtenu de Rome la permission d'exercer la médecine à l'Hôtel-Dieu : voilà les principaux titres de celui que Dom Jamet appelle si justement « le bon M. Souart »

Je ne puis ici que donner un aperçu de la longue carrière canadienne de M. Souart. Elle commence le 12 août 1657. Ce jour-là, le jésuite Claude Pijart, qui faisait les fonctions de curé depuis 1650 et dirigeait Marguerite Bourgeoys depuis 1653, cède la place au sulpicien Souart. On quitte le Fort. La "maison de Mlle Mance " sera le presbytère, comme la chapelle de l'Hôtel-Dieu - en attendant la première église Notre-Dame (1683) - continuera de tenir lieu d'église paroissiale. C'est là que notre premier curé sulpicien - humble curé de campagne - écrit ses a actes" officiels : baptêmes, mariages, sépultures, dans le "Premier registre de Ville-Marie», commencé en 1642, que nous possédons encore. Rien d'émouvant comme d'y retrouver, plusieurs années de suite, sa signature (il signe son nom avec un tréma sur l'u) et celle des personnages prestigieux de ces temps héroïques : Paul de Chomedey (il signe toujours ainsi), Jeanne Mance, Marguerite Bourgeoys, Lambert Closse, Charles Le Moyne, Jacques LeBer et combien d'autres... Le 21 novembre 1657, le curé et M. de Maisonneuve procèdent à l'élection des premiers marguilliers. Le 22 janvier suivant, le curé, le vicaire (M. Galinier) et les marguilliers signent l'acte par lequel Maisonneuve donne à Marguerite Bourgeoys le "colombier *, berceau de la Congrégation Notre-Dame. En 1662, il concède aux Hospitalières cent arpents de terrain, qui viennent s'ajouter aux autres cent déjà concédés par M. de Maisonneuve en 1659. En 1664, au nom du Séminaire de Paris, il prend possession de la seigneurie de Montréal. En 1665, il reçoit la profession de Marie Morin, la première religieuse canadienne à Montréal et le premier historien canadien de Montréal. En 1666, il cède la cure à $M$. Gilles Pérot et ouvre enfin son école de garçons, qui en groupe déjà soixante-treize, dont les âges s'échelonnent entre cinq et douze ans. Il assiste, en 1672 , à la pose de la première pierre de la future église Notre-Dame. De 1674 à 1676, il est de nouveau, cette fois après M. Dollier de Casson, supérieur du Séminaire, juste à temps pour donner à Marguerite Bourgeoys le a terrain et emplacement " où elle pourra, après une douloureuse attente de dix-huit ans, bâtir sa chapelle de Bon-Secours. Sœur Bourgeoys, il la connaît depuis près de vingt ans et jamais il n'a cessé d'être son protecteur et son appui. Lorsqu'il l'appelait "la petite sainte Geneviève du Canada ", il ne faisait pas seulement allusion à sa petite taille; il exprimait aussi son estime et son admiration pour l'hérö̈que fondatrice. Il n'a pas moins de sympathie pour la personne et l'œuvre de Jeanne Mance et pour les Hospi- 
talières de l'Hôtel-Dieu. Ainsi affirme-t-il, sans peur et sans faiblesse, sa fidélité à la pensée de M. Olier et aux desseins des Fondateurs de Montréal. Il a vraiment été a le bon M. Souart "! Après 1676, il ne conserve plus que le poste d'aumônier de l'Hôtel-Dieu et entre dans une sorte de retraite. Bien à tort, il se croit en disgrâce. Il demande à rentrer en France. Monsieur Tronson lui écrit plusieurs lettres et l'encourage à patienter. Finalement, il le rappelle en 1688. Monsieur Souart mourra trois ans plus tard, à quatre-vingts ans.

A cette date de 1691 , il y a déjà vingt ans que M. Galinier est mort. Au registre des sépultures, on lit ces lignes : "Le vingt octobre mil six cent soixante et onze a esté enterré Messire Dominique Gallinier (sic) de la communauté des prestres de ce lieu âgé de cinquante cing ans. Natif de la conté de Foys (sic). M Mirepoix, lieu d'origine de M. Galinier, faisait partie de l'ancien comté de Foix, aujourd'hui compris dans l'Ariège. Des rares détails que nous avons sur lui, j'en citerai deux. Après la mort tragique de MM. LeMaître et Vignal, M. Souart et son vicaire se retrouvent seuls. Au début de juin 1662, le père Chaumonot, jésuite, vint, de Québec, leur prêter main-forte. Pendant quatorze mois, ils travaillèrent tous trois à établir la Confrérie de la Sainte-Famille. On logeait alors au séminaire récemment bâti par MM. LeMaître et Vignal. Le père Chaumonot écrit, dans son autobiographie, qu'ils vivaient " dans une si parfaite union qu'on les aurait pris, eux pour être de la Compagnie de Jésus, et lui pour être du Séminaire de Saint-Sulpice ». En 1658, Marguerite Bourgeoys décida d'accompagner Jeanne Mance en France. Elle remplissait alors l'office de sacristine à la paroisse et y tenait beaucoup. Monsieur Galinier lui permit de partir, à la condition expresse - il voulait sans doute taquiner sœur Bourgeoys - que son absence ne durerait pas plus d'un an. Partie le 29 septembre 1658, Marguerite Bourgeoys était de retour le 29 septembre suivant, jour pour jour et « à peu près heure pour heure», disent les Ecrits autographes. Et elle garda sa sacristie !

Lorsque M. de Queylus retourna en France, le 22 octobre 1659, M. d'Allet ne put l'y suivre, retenu à Québec par une maladie qui dura tout l'hiver. Une fois rétabli, il regagna Montréal, à la fin d'avril 1660, et se livra au travail des missions. Sa santé ayant de nouveau fléchi en 1664, il rentra en France. Lors du dernier séjour de M. de Queylus au Canada (1668-1671), M. d'Allet l'accompagna de nouveau et retourna définitivement avec lui. Dans son Histoire Littéraire de la Compagnie de S.-Sulpice, L. Bertrand le range parmi les a auteurs sortis de la Compagnie ». On ignore la date exacte de sa mort, mais il vivait encore en 1693. Un peu perdu dans l'ombre de son grand patron, $M$. d'Allet s'est quand même acquis une sorte de célébrité par des Mémoires sur ses voyages au Canada. Faillon réfère quelquefois à ces Mémoires, 
qui ont eu la singulière destinée d'être publiés dans les Euvres de Messire Antoine Arnauld, le célèbre janséniste, grand ennemi des Jésuites ... et des Sulpiciens!

Deux autres noms ont été mentionnés au cours de cette brève étude sur les débuts de Saint-Sulpice à Montréal : Gilles Pérot et Dollier de Casson. L'importance du rôle joué par ces prêtres m'oblige à vous livrer, en terminant, quelques notes à leur sujet.

Monsieur Gilles Pérot, originaire du diocèse de Chartres, arrive en juillet 1665. Monsieur Souart lui cède la cure à l'automne de 1666. Curé-missionnaire, comme M. Souart, jusqu'en 1678, il devient alors le premier "curé d'office", au nom du supérieur, et occupe le poste jusqu'en juillet 1680. Le 9 octobre 1667, il réunit au Séminaire un groupe de paroissiens pour adresser au roi Louis XIV une supplique, à l'effet que celui-ci accorde des lettres patentes à la Congrégation des Institutrices de Ville-Marie. La supplique est signée par lui et par le juge royal. Sous la direction de M. Dollier de Casson, il commence la construction de l'église Notre-Dame et, dans le même temps, encourage Marguerite Bourgeoys à reprendre son chantier de Bon-Secours. La chapelle est prête en 1678, cinq ans avant l'église Notre-Dame. Le 17 juillet 1680, alors qu'il se préparait à célébrer la messe, il meurt, frappé d'un coup de sang.

Monsieur François Dollier de Casson, né en 1636, au diocèse de Nantes, séjourna au Canada de 1666 à 1674, puis de 1678 à 1701. Il fut supérieur du Séminaire après M. de Queylus, de 1671 à 1674. Puis succédant à M. Souart au même poste, en 1678, il demeura supérieur jusqu'à sa mort, arrivée en 1701. Un homme extraordinaire, un vrai "géant ", au propre et au figuré : ancien soldat, aumônier des troupes, explorateur, missionnaire, architecte, constructeur, ingénieur! C'est lui qui construisit la première église Notre-Dame. A titre de seigneur de Montréal, il fait homologuer les voies publiques existantes, baptise les rues de ce qu'on appelait alors "la haute ville ", entre les rues SaintPierre et Saint-Gabriel, commence le creusage du canal de Lachine, écrit, sous forme de lettres annuelles couvrant la période de 1641 à 1672, l'Histoire du Montréal. Avec M. Olier, M. de Bretonvilliers, M. de Queylus et M. Souart, il compte parmi les plus généreux bienfaiteurs de la colonie.

Jean LANGIS, p.s.s., L.Th.

Secrétaire provincial

Prêtres de Saint-Sulpice Montréal, P.Q. 